\title{
Structure: Its Shadow and Substance ${ }^{1}$
}

\author{
Bas C. van Fraassen
}

Views of science and of nature change hand in hand. In the seventeenth century the new sciences inspired a hard-line ascetic metaphysics. Theorizing, the new scientists stripped the world of its appearances, its qualitative riches, leaving res extensa as the sole reality of nature, veiled before the mind in its sensory illusions. New instruments such as the microscope promised to confirm the theories directly, and the newly schooled mathematical imagination promised to represent reality as fully intelligible to the mind.

But as science moved on to new conceptions of nature, its self-image changed as well. Here I shall try to follow two lines of retrenchment as physical theory lent itself increasingly less to naïve realism: reification and structuralism. Within these, I will focus specifically on the new structural realism, which recently tried to position itself between scientific realism and instrumentalism. After examining its difficulties, I will offer an empiricist view in response to the same ambition.

Planck Against The Heretics

On December 9, 1908 Max Planck addressed the Student Corps of the Faculty of Natural Sciences of the University of Leiden. His announced topic was The Unity of the Physical World-Picture, but the real intent was a polemic against a whole bevy of famous scientists who had turned against realism in the past fifty years. These misguided heretics included Maxwell, Boltzmann, Hertz, and most of all Ernst Mach, who was to be Planck's main target in this lecture. In Planck's eyes they had forsaken the faith of their fathers. He speaks against them with passion: 
When the great masters of exact research contributed their ideas to science: when Nicolaus

Copernicus tore the earth from the center of the universe, when Johannes Kepler formulated the laws named after him, when Isaac Newton discovered general gravitation, when your great countryman Christian Huygens put forward the wave theory of light, and when Michael Faraday created the foundations of electro-dynamics ... [Mach's] economical point of view was surely the very last thing which steeled the resolve of these men in their battle against traditional views and towering authorities. Nein! ... it was their rock-solid belief in the reality of their world picture.

(Planck 1909/1992, p.131)

But there is some irony in this episode. Planck had not exactly been unaffected by the heresy he is attacking. Both in this passage, in his title, and indeed throughout his lecture, he speaks of physical theories as pictures and of the product of science as a whole as a world-picture. This language of 'science as representation' was the common coin of his opponents, and indeed, the notion and name of world-picture is attributed to Hertz (cf. Blackmore). When Planck says that this heresy "enjoys great popularity, particularly in circles of natural scientists" (ibid. p. 129) he bows to it in his own choice of language, while arguing against it. For Planck considered this heresy to be a mistaken if understandable response to the "unavoidable disillusionment" when the mechanical world view began to disintegrate.

So what was that popular philosophy? Planck directs himself primarily against Mach, but it is in Boltzmann's more moderate and less philosophical writings that we see the story better.

\section{Boltzmann, his story}

Boltzmann presents his own point of view as deriving mainly from Maxwell and Hertz, two of the heroes of recent achievements in electromagnetism. Maxwell's writings are not exactly unambiguous. In fact he is for the main part taken as believing in the reality of the ether and of the electromagnetic waves in the ether, while sometimes despairing of any purely mechanical theory of their character. However, as Boltzmann emphasizes, Maxwell speaks of the envisaged mechanisms as merely analogies, partial analogies, that allow us to get an imaginative grasp on the equations. The equations must on the one hand fit the observed magnetic, electrical, and optical phenomena, and on the other hand allow of some understanding of 
the theory as a description of a physical process. But on that second point we receive mainly analogies with other forms of material propagation, diffusion, and interaction -- with gases, fluids, and heat. Maxwell himself cautions us against thinking of this as a true description of reality behind the phenomena:

By a judicious use of this analogy [between Fourier's equations of heat conduction and the equations of the electrostatic field] ... the progress of physics has been greatly assisted. In order to avoid the dangers of crude hypotheses we must study the true nature of analogies of this kind. We must not conclude from the partial similarity of some of the relations of the phenomena of heat and electricity that here is any real similarity between the causes of these phenomena. The similarity is a similarity between relations, not a similarity between things related. (Maxwell 1881, pp. 51-52)

Then, as Boltzmann sees it, Hertz makes a virtue of necessity and asserts this as a way to understand the scientific enterprise as a whole. Thus Hertz writes and Boltzmann cites:

scientific accuracy requires of us that we should in no wise confuse the simple and homely figure, as it is presented to us by nature, with the gay garment which we use to clothe it. (Hertz 1893/1962, p. 28)

Indeed, with Hertz we begin to have such an emphasis on the representations and their adequacy to the experimental facts as sole anchor, that we can quite understand Planck's sense that the represented world is mostly counted as well-lost for love of theory:

We form for ourselves inner pictures or symbols of external objects; and the form which we give them is such that the necessary consequences of the pictures in thought are always the pictures of the necessary consequences in nature of the things pictured ....

The pictures which we here speak of are our conceptions of things. With the things themselves they are in conformity in one important respect, namely in satisfying the above 
requirement. For our purpose it is not necessary that they should be in conformity with the things in any other respect whatever.

(Hertz 1894/1956, pp. 1-2; see also p. 177)

Boltzmann, lecturing on this in 1899 , expressed the philosophical point of view most trenchantly:

We know how ... to obtain a useful picture of the world of appearance. What the real cause for the fact that the world of appearance runs its course in just this way may be; what may be hidden behind the world of appearance, propelling it, as it were -- such investigations we do not consider to be of the task of natural science.

(Boltzmann 1905a, p. 252)

Mach's reaction

Finally, we may note Mach's reaction to Planck's criticisms of this heretical train of thought. Just as Boltzmann does in this last passage, so Mach atttibutes those realist misgivings to metaphysical dreams by which philosophers have infected physicists from time to time:

In any case, physicists have nothing to seek "beyond the appearances". Whether philosophers will always find it necessary to affirm something real ... whose relations may only be recognized in the wholly abstract form of equations, may be left entirely for the philosophers to decide. [...] Hopefully, physicists of the $20^{\text {th }}$ century will not let their investigations be disturbed by such meddling!

(Mach 1910/1992, p. 124-125)

So, as we see here, Planck and Mach each depict the other as having strayed from the true concerns of natural science into a mistaken philosophical conception of their common enterprise.

These passages are revealing, but they are more polemical than instructive. We need to see precisely what options were coming into play. 
Poincaré On The Meaning Of Maxwell's Equations

As focus I will take Poincaré's verdict on classical electromagnetism: Maxwell's theory is [just] Maxwell's Equations. This verdict was also a sort of capitulation. Maxwell himself had attempted to prove the existence of models of his equation in mechanics. The theory of the ether was a sustained attempt to provide them with a concrete mechanical underpinning (cf. Stein, pp. 61-62). When Maxwell had his theory fully worked out, he discarded the earlier rather primitive ether models but tried to subsume his theory under the generalized dynamics of Lagrange, which deals with mechanical systems whose internal constitution is not fully specified. ${ }^{2}$ In Poincaré's verdict we recognize a definitive goodbye to the interrelation of matter and ether as a live topic in physics. ${ }^{3}$

Poincaré's views on science were generally tending toward what Planck considers the great heresy, though stated with caution and diplomacy. In the spirit of Hertz he speaks of "images we substituted for the real objects which Nature will hide for ever from our eyes. The true relations between these real objects are the only reality we can attain ...." (Poincaré p. 161) Of the principle of conservation of energy, for example, he writes that if we try to enunciate it in full generality, "we see it vanish, so to speak, and nothing is left but this -- there is something which remains constant...." (p. 132).

But Poincaré's verdict was paradoxical and provocative. If science describes nature, Maxwell's Equations must form a theory about what something is like. Mustn't the theory also say what that something is?

Two responses: reification and structuralism

If Maxwell's Equations are statements, the question is what they say. If they are not statements, the question is how they can amount to a theory at all.

If we leave aside the more instrumentalist (non-statement) options, we detect here two not very well distinguished alternatives. The first is that yes, it is the electromagnetic field itself, which is a thing, and not the shape or form of something else. Today that is an often expressed view, perhaps not always clearly distinguished from rejection of the classical ether: "Fields in empty space have physical reality; the medium 
that supports them does not" (Mermin, p.753). There is no puzzle, just a new ontology, some new and previously inconceivable furniture for the world. I'll call this alternative reification.

The second alternative is a little more agnostic. It could be expressed like this:

The Equations only describe a form or structure -- if that is the form or structure of something, that is an unknown entity. The field is first of all an abstract entity (mathematical: e.g. a function assigning values to points in space), though we can of course also give the name "field" to whatever it is -- if anything -- that bears this structure.

That unknown bearer might well have other properties, just as ordinary things have properties beside their shape. But the theory does not describe those. Science abstracts, it presents us with the structural skeleton of nature only.

To begin this sounds rather reactionary, just when we have discarded the ether and its frustratingly elusive qualities. But there is an often mentioned bit of support. Important equations tend to recur in many places. They tend to identify recurrent patterns in nature, found not once but many times. Often a new process is first described in analogy to an old one, with the equations transposed or reinterpreted. Heat diffusion and gas diffusion are analogous, the harmonic oscillator crops up everywhere.... So the equations omit the distinguishing characteristics.

As a reason for structuralism, this observation does not show much at all. For whenever we see the same equations describing two scientific subjects, we also see science describing the differentiating characteristics. If we didn't, we wouldn't have an example to give! The point that such equations describe at once many different processes needs serious reflection, but it is not much of an argument for anything here.

So there must be other reasons why both scientists and philosophers have kept returning to this sort of view. It has taken various forms:

moderate structuralism: the theory describes only the structure of a bearer, which has also nonstructural features (though science is said not to describe those) radical structuralism: "structure is all there is" 
in-between structuralism: the structure described by science does have a bearer, but that bearer has no other features at all.

What I presented initially is therefore the moderate form. The radical form, to which we will pay special attention below, will not be all that easy to grasp. Finally, I simply interpolated the intermediate form between them; I have not seen this discussed in philosophy of science, but only in more purely metaphysical disquisitions.

The currently fashionable term, which still covers various versions, is structural realism. To speak of all the varieties at once I'll say structuralism [about science].

Planck's diagnosis contested

When Planck reflected on how the great heresy could have taken hold among his colleagues, he diagnosed it as the counsel of despair. They turned toward anti-realism and empiricism only after classical physics had run into severe difficulties, and the physics community had gone through a slough of despond.

As I will now try to show, that is not so. The very same philosophical options can be seen to emerge much earlier. Indeed, they emerge very naturally when science proves itself too complex for philosophical naiveté. We see a clear tendency to reify whatever theories invoke in their representation of nature. But conceptual difficulties and the increasingly mathematical character of science foster the structuralist impulse. As interpretations then threaten to become lost in "a bloodless ballet of categories", however, weariness sets in, and the pendulum swings back to reification. Eventually we must find some point of stable equilibrium!

\section{On The Road To Structuralism}

Let's begin with the disparity the scientific image and the manifest image in the17th century. You can easily see how structuralism could have been a first response. Perhaps, Galileo's contemporaries might have said, the scientific image represents only some aspects of the real and manifest world, leaving many other real aspects out of account. Then, later on, if that idea were accepted, one could add: the aspects represented by science together constitute the structure or form of the world, while all the rest is the 'matter' or content. That reaction, which would not sound all that radical, would thus be to say: fine, science abstracts, 
it ignores everything else -- it will be useful to find the structural patterns and ignore the rest for a while. We are reminded here of Cardinal Bellarmini's advice to Galileo of how to view the Copernican system. One imagines that Bellarmini would wish Galileo to think no better of his 'new sciences' in general.

Galileo himself, and the mechanical philosophers generally, were more radical. They rejected any such moderate structuralism in favor of reification of their world image. They said, No, THIS is all there is to it. Atomism would supply the resources to extend mechanics into a comprehensive, complete, universal physics. Colour, smell, sound, all perceptual qualities would be reduced to combinations of primary qualities in the things and sense organs.

We should place this more radical metaphysical view in contrast to a methodological point that everyone can agree upon. Galileo's restriction of science to primary qualities was a much needed disciplinary move. Aristotelian scientists had felt free to multiply theoretical terms for so-called occult properties. These were a thing's properties that can't be explained on the basis of its composition. Galileo's discipline was to determine beforehand a small set of properties and restrict scientific descriptions to those. Not coincidentally, of course, they were the properties representable by geometry and arithmetic: number, size, shape ....

The contrasting metaphysical thesis was extremely speculative, but for a while it looked as if the path to reduction would be pretty short. The microscope especially raised great hopes. Atoms would soon be visible, and the reduction of sensible qualities to mechanical affections would be observable. Color did seem more of an illusion when everything looked just black, white, and gray through those lenses -- the argument is not exactly airtight, to say the least, but lent some plausibility to that supposedly really real world of colorless atoms.

The microscope

I am not exaggerating. Around the middle of the seventeenth century these were the expressly emphasized hopes of vindication for the mechanical philosophy. ${ }^{4}$

One writer on the microscope, Charleton, remarked that of course the ancients who lacked optical instruments would have greeted the atomic hypothesis with scepticism -- but our situation is quite different! Robert Hooke writes in the preface of his Micrographia (1665) that with the aid of the microscope we will discover 
the subtilty of the composition of Bodies, the structure of their parts, the various texture of their matter, the instruments and manner of their inward motions, and all the other possible appearances of things .... we may perhaps be inabled to discern all the secret workings of Nature, ... manag'd by Wheels, and Engines, and Springs ....

And Hooke's contemporary Henry Power's Experimental Philosophy (1664) says in its preface: We might hope, ere long, to see the Magnetic Effluviums of the Lodestone, the Solary Atoms of Light ..., the springy Particles of Air, the constant and voluntary motion of the Atoms of all fluid Bodies, and those infinite, insensible Corpuscles which daily produce those prodigious (though common) effects among us.

Notice that the optical microscope will reveal even the composition of light! Besides this radical misunderstanding of what the microscope can do, several other errors both of fact and of principle would soon become evident.

\section{Mathematization of the World Picture}

In a way, it was the mathematization -- rather than mechanization -- of the world picture was the glory of 17 th century physics. Descartes' physics could leap forward because he created analytic geometry. Newton's could, because he created the calculus. So you would not expect any of them to complain of too much mathematics. But they do. They remember Bellarmini and other Aristotelians charging astronomy with mere mathematical recasting of the phenomena, and with failure to provide true physical content. But now the Cartesians repeat that charge, mutatis mutandis, against Newton. To see their complaint we must remember that the Cartesians were true adherents of the mechanical philosophy, to which the English still paid verbal tribute.

We see the primal form of that view of nature most clearly in Descartes' posthumous treatise The World, or Theory of Light. This magnificently ambitious work initiated the entire Faustian project of modern 
theoretical science. To begin, Descartes says disingenuously that he wishes to set aside all real physical and empirical questions. He only wants to attempt the mental construction of a world entirely intelligible to the human mind. The result will be precisely the world God would have created if it was going to be a world entirely transparent to human reason, with no mystery in it at all. This World we can clearly understand, for its image, here provided, is the mind's own product. ${ }^{5}$

Of course, a little later in the treatise Descartes announces that this constructed World of his matches everything we have found out about the real world. That is precisely the scientific success he aimed for. And finally he adds the reification of his image -- this perfectly intelligible World, he says, is the real world.

In all this we may see a lasting, paradigmatic project for theoretical science. Construct a world entirely transparent to the human mind; then show that all known phenomena fit therein. But of course, Descartes' own version had belonged to his own time. Most specifically he counts only clockwork mechanism as entirely intelligible. The World must be a pushme-pullyou machine, if we are to understand it completely.

But now comes Newton, whose success is much greater by the same general standard; but who has introduced non-mechanical elements, namely forces which act at a distance, instantaneously. For with Newton, despite much lip service, the early mechanical philosophy bit the dust. The introduction of these forces is a major innovation. The world is no longer a clockwork, in which everything proceeds by push and pull. For Newton himself, this was serious; he was reared in the mechanical philosophy and did not at all see himself as overturning it. He felt very deeply the Cartesian charge that his masses, absolute velocities and forces acting instantaneously over any distance, holding the universe together in a curiously harmonious symphonic whole, reintroduced the occult properties that his own scientific heroes had just banished. Thus Newton defended his own achievement in words which indicate that he hopes for a truly mechanical explanation of gravity. But over the next century, the victorious Newtonians discarded those scruples, and began to think of Newton's own models as the fulfilment of the mechanical philosophy's aspirations.

What do the Cartesians think of Newton's success? What they see is the replacement of a humanly intelligible, concrete description of nature by mathematical abstractions. Except that they themselves are eclipsed during the next century, what they see stands out ever more clearly. 
The Newtonian paradigm tells scientist to describe patterns of motion, velocity, acceleration, by the new techniques of the calculus, and to add an assignment of masses and forces which turns this pattern into something that satisfies Newton's laws. Very soon they constrain themselves to assign central forces and to conservation laws -- under these constraints, success is not trivial. But the success is a triumph of mathematics, and mechanical action by contact is most definitely not among the constraints. Thus the reification of the World constituted entirely of entities characterized solely by 'mechanical' parameters sounds more and more like a hollow addition to the mathematics -- a vanilla story, as we call that now.

Signs of disillusionment

The gap between the manifest and scientific images had certainly widened -- and had become salient by the end of the 17 th century. That is why we see a general disillusionment with the microscope early in the eighteenth. Newton distinguished carefully the apparent motions described by our measurement results and observations from the reality he postulated. Berkeley and Locke then point out that instruments won't help with the question of principle, and will certainly not reveal a reality behind the appearances.

Later scientific realists seem not to recall this insight of Locke and Berkeley. But it remains crucial to appreciate their point if we are to understand the continuing dialectic. By means of instruments we create new phenomena. Whatever will become visible through optical or any other instruments will still be only those apparent motions Newton described as observable -- not the forces, masses or other explanatory characteristics behind those appearances. The new appearances experienced by the inquiring and phenomenon-creating scientist must also be saved - but do not reveal the hidden theoretical underpinnings directly. All we will ever get are appearances, all we ever get are more and novel observable phenomena to save, even if conjured up by previously unimagined instruments.

How are those appearances saved? By fitting them into the postulated theoretical world. In Newton we see a constant vacillation between our two main options. When in a triumphant mood, he asserts his law of gravity to describe a new, real cause -- satisfying his vera causa Rule. When pressed and conscious of the Cartesian complaints, he says instead that he will frame no hypotheses, and that it is no mean achievement to have discovered a general form or pattern for planetary and projectile motions. This is just the opposition with which I began, between reification and structuralism. 
The 19th/20th century

Maxwell repeated Newton's philosophical quest in one sense, Poincaré in another. It is as if a great pendulum is swinging back and forth between two extremes. On the one end, appearances are illuminated by being fitted into a theoretical structure; on the other, far end of the swing that structure itself is held up as the sole reality, the substance of the world. ${ }^{6}$

When Maxwell thought about the ether, he would have been happy to find a Newtonian 'mechanical' model. ${ }^{7}$ Things had gotten worse still, for no such model was forthcoming. The ether, finer than air but more resilient than steel, just would not comply. And yet we see that in this case too, the success of the new theory vanquished all scruples. The newer sense is that certainly there is a process whereby the electromagnetic waves are propagated in space -- and Maxwell's equations are what describe this process, as fully as is required. Newton and Maxwell, in their day, agonized and vacillated between reification and structuralism -but for their heirs the question disappears. Their agony was then seen only as the aftermath of an oldfashioned world view.

That is one of the main reasons why, I think, we see the structuralist reaction emerging in the $19^{\text {th }}$ century. As so often happens, what is earlier seen as a failure or shortcoming becomes the glory of a new generation. When Kirchhoff, Mach, and others attempt to define mass and force, what they really want to do is say: mechanics describes only the general, mathematical structure of motion, but that is precisely the wonder of its success. Mass and force are only theoretical parameters. We know now, through the evolution of mathematics, that abstract structures of the same type should be thought of that way. (That is, we describe a structure with some parameters left undetermined, but we place constraints on those parameters; the different admissible values of those parameters identify the instantiations that can occur in nature.)

By the end of the $19^{\text {th }}$-century, this view of mechanics is expressed in a much more general form for all of physics, all of science, by prominent physicists. I'll just cite here the view as expressed later by Herman Weyl:

If nature were all lawfulness then every phenomenon would share the full symmetry of the universal laws of nature. [...] The mere fact that this is not so proves that contingency is an essential feature of 
the world.... The truth as we see it today is this: The laws of nature do not determine uniquely the one world that actually exists, not even if one concedes that two worlds arising from one another by ... a transformation which preserves the universal laws of nature, are to be considered the same world.

(Weyl 1952, pp. 26-27.)

Knowing a great deal about Herman Weyl, we can easily detect what is in his mind. He is pre-occupied with the picture of a group having a number of non-isomorphic irreducible representations.

But we can illustrate his point in a simple way. The point he is making is that not everything left invariant by the transformation group that corresponds to a law of nature is itself a matter of law. As example we can take classical mechanics, where velocities are not invariant, they are relative to frames of reference. So the mechanics really does not describe patterns of velocity, but rather of acceleration, which is invariant. Yet we cannot very well think we have to do here with only one pattern, even if we ignore all that is framerelative. For number too is invariant, but is left entirely unspecified and unconstrained in the laws of motion. The number of bodies that make up the system is invariant, but it is not a matter of law. Kepler had indeed held that any adequate physics would have to explain why there are exactly six planets. Happily Newton left number unspecified, so that his laws are instantiated in N-body systems for every natural number $\mathrm{N}$.

By the end of the $19^{\text {th }}$-century the mathematization of the physical world picture was nearly complete. Is Hertz' mechanics a theory of physical systems or of mathematical structures?

Hard to say. It becomes even harder to say when the theory of gravity becomes the theory of solutions of the GTR field equations, and atomic physics the theory of solutions of Schroedinger's Equation. I exaggerate of course, and I ignore momentarily what's nearest to an empiricist's heart -- experiments, observations, empirical phenomena investigated or created, enlarging the realm of experience to previously unimaginable breadth. But it is the theorists who present us with the scientific image of the world. And these theorists write the Book of Nature in the language of mathematics.

Thus, by the end of our century, the whole world complains to the scientist in precisely the words of the Cartesian to the Newtonian. Are you describing nature, or just putting it into a beautiful mathematical format? The implied complaint is not about empirical adequacy. Certainly Newtonian science improved the 
tables of tides as well as ballistics, predicted the flattening at the poles, the return of comets.... Twentiethcentury physics gave us transistors, fission, fusion, radar and laser optics. But what happened to the promise of a World entirely, thoroughly, and completely transparent to the human mind? ${ }^{8}$

The New Structural Realism

Modern science began with Galileo's and Descartes' evangelical reification of the scientific image of the world. In some of Newton's more defensive remarks we can already see a vacillation between two responses. On the one side we see that sort of realism according to which every theoretical posit -- and nothing else -- corresponds to an element of reality. On the other emerges the more moderate structuralism which attributes to science success in abstracting important patterns in nature only, and describing those. Two centuries later the structuralist impulse has become very salient and erupts in such dicta as Poincaré's about Maxwell's equations.

From scientific realism to structuralism

Contemporary philosophy of science displays a similar pilgrim's progress. Scientific realism begins with the intuition that the empirical success of science cannot be just a miracle or a coincidence, but must have an explanation. This requirement for explanation is crucial to both the motivation and content of scientific realism. However, the explanations realists offer are not all the same. Here is a more or less central version of the view, as I see it:

The aim of science is to provide us with a literally true story about what there is in the world, and this aim is actually achieved to a great extent, because it is pursued by effective means to serve that end.

By themselves, do these contentions explain the empirical success? Certainly not! To explain the empirical success of science, something must at least be added about to what extent, and in what respect, that aim of true description is achieved. Suppose, for instance, that the extent to which that aim is achieved is only this: our theories now make true predictions about all the observable phenomena they deal with. That would 
certainly be a great extent! But it would be no more than the very success which the realists require to be explained..$^{9}$ So what additions do the realists introduce?

The standard first addition was that at least the theoretical entities postulated by science are real. Galileo was quite right that there really are atoms, though he was entirely wrong about what they are like. The truth of the atomic hypothesis supposedly explains much of scientific success since then.

The drawback to this idea appears immediately when we apply it to the ether. Shouldn't the question What explains the success of this successful theory? receive the same answer in each case, once this 'standard addition' to the realist view has been made, precisely on the basis that it provides the resources to answer this question? But if so, the success of theories of light, electricity, and magnetism in the $19^{\text {th }}$-century would then be explained in part by the reality of the ether. Today we grant the empirical successes, but can hardly explain them that way! Of course, we can now provide the different explanation, that the theories happened to track certain empirical regularities, and not any reality behind them. But if that is a good explanation now, why not then also? And if it is always a good explanation, the basis for this realist addition disappears. On the other hand, if it does not count as a good explanation, then the realists have not done what they themselves demand here: to explain the success of science in general. For such examples can be multiplied, especially when we realize that the same words -- such as "atom", "electron", "field" -- will later be used for entities quite different from any they originally stood for (so that when they are retained, the reality of their later referents does not explain the success of the earlier stages of the theory).

Thus scientific revolutions, and even evolutions, embarrass the standard scientific realist. The standard pattern of realist explanation of empirical success has become a historical embarrassment, for its instances are by now expected to fail. This was forcefully pointed out by John Worrall. But Worrall insisted that despite all this, we must have a realist explanation of the success of science:

The main argument for scientific realism is that our present theories in science are so successful empirically that they could not have gotten that way by chance -- instead they must somehow have latched on to the blueprint of the universe. The main argument against scientific realism is that there have been enormously successful theories which were once accepted but are now regarded as false.... [T]here is [a] reasonable way to have the best of 
both worlds: to give the argument from scientific revolutions its full weight and yet adopt some sort of realist attitude towards presently accepted theories in physics and elsewhere -through structural realism, a position adopted by Poincaré, and here elaborated and defended. (Worrall 1989, Summary)

Worrall's solution was to say that we must make a different addition to the initial explanation schema. What science succeeds in first and foremost is not the identification of real things in nature that make up the fabric of manifest phenomena. Instead, science succeeds in discovering the structure of whatever it is in nature that bears these appearances. That je ne sais quoi underlying the phenomena becomes partially manifest in structure while remaining forever unknown in content or quality.

Thus Maxwell's Equations are retained because they describe a structure which is really there, although any additional description has to be discarded.

This was a new move for scientific realists in the second half of the $20^{\text {th }}$-century. It was not of course actually new in either philosophy or science. As we saw, at the beginning of the $20^{\text {th }}$ century this was salient doctrine. Bertrand Russell gave a striking analogy. What do we know of the world behind the phenomena? Listen to the radio, and hear the sounds which were produced in the studio many miles away. In between are the radio waves which have none of the qualities of sound. But we infer they must have structure which encodes the structure of this sound. Thus we know a great deal about those radio waves on the basis of observation: not what qualities they have or what they are like in themselves, but their structure. And it's precisely that, and only that, which science describes (as it happens, of course, what Maxwell's Equations describe).

Scientific revolutions provided the great argument against the view that science is a steadily accumulating store of knowledge. The theoretical furniture of the world is continually discarded, removed, and replaced; certainly not accumulated. But now Worrall tells us that there is after all an accumulating store of knowledge: more and more knowledge of the structure of that furniture, although ideas which go beyond structure fall to the knife at every step.

A very pretty idea. It is regrettable only that the division between structure and content is never discernible beforehand. The structure discovered is identifiable only in retrospect -- it is the part retained 
through scientific theory change .... The atoms are still there at some level, so that was structure. The ether is no longer there, at any level, so that was a mistake about content....

Yet the structural realist literature and the earlier structuralisms about science as well, despite their flaws, retain genuine appeal. Surely there $i s$ an accumulation of knowledge about nature in science, and surely that is the key to its success. What can we make of this?

The Ladyman variant: objectivity and invariance ${ }^{10}$

James Ladyman (1998a, 1998b) argues that the guiding idea for structural realism was equivocal between two positions. These positions are sharpened forms of what I called reification and radical structuralism. Ladyman sharpens these into:

an epistemological position: 'ordinary' scientific realism plus the view that all we can come to know (have reason to believe, can hypothesize?) about is the structure of nature alone.

an ontological position: that the structure of nature described by scientific theories is really -properly understood -- all there is to nature.

The former requires that sense be made of two dichotomies ${ }^{11}$ :

epistemic dichotomy: what we have epistemic access to (can come to know, can theorize about in a scientifically significant way) and what we have no such access to. ontic dichotomy: the structure of nature and those features which are not structure (matter or content)

We certainly have examples of views for which we can draw such distinctions. Here is a main example. The quantum mechanical description of an assembly of particles of the same type ( a fermion gas, for example) includes nothing to individuate or differentiate these particles from each other. One option was to postulate 
individuating factors (thisness, haecceity) which do not enter the quantum state -- an empirically superfluous hidden variable. Another option was to say that this very question reveals an inadequacy: the proper formulation will be in terms of fields, denying the existence of individual or individuatable particles. A still further option was deny the identity of indiscernibles, and say that these particles are distinct without being in any way different. ${ }^{12}$

This 'problem of identical particles' and the options it allows for interpretation can be used as paradigm to motivate Ladyman's exploration, following Weyl, of invariance as definitive clue to structure. In the above 'identical particle' debate, the salient scientific fact is that the quantum mechanical state is permutation invariant. This may be glossed as :

only those features which are permutation invariant belong to structure -- if there are other features they belong to 'content'.

Any such other features there may be characterize what bears that structure, in a way which goes beyond what science describes.

Reading the scientists who write about this, we certainly see ample precedent for this gloss, though it remains generally quite unclear which options are being taken up. Thus Weyl:

Objectivity means invariance with respect to the group of automorphisms.

(Weyl 1952, p.132; cited Ladyman 1998b, p.237)

Which topic and it consequences Weyl places in historical perspective:

the founders of modern science [...] discarded the sense qualities, on account of their subjectivity, as building material of the objective world which our perceptions reflect. But they clung to the objectivity of space, time, matter, and hence of motion and the corresponding geometric and kinematic concepts [....] But soon the objectivity of space and time also became suspect. (Weyl 1931/1950, p. 17; cited Ladyman1998b, p. 237) 
But this is treacherous ground, for "objectivity" has many antonyms. ${ }^{13}$ "Objective" can contrast with "subjective", "relative", perspectival", "unreal" and "scientifically insignificant". Thus, if electrons have 'haeccity' to individuate them, that is not invariant, hence (pace Weyl) not objective -- but it is neither subjective, nor relative, nor unreal in that case. On the other hand, length (being not invariant under Lorentz transformations) is relative, but not subjective, unreal, or even scientifically insignificant. And so forth. ${ }^{14}$ Let me suggest a specious argument, of which no writer on this subject is guilty, but which might lurk behind an unsuspecting reader's comprehension:

A symmetry of an object is a transformation that leaves the object the same -- identical to itself. The paradigm examples are reflection through an axis of bilateral ('mirror image') symmetry, or a rotation through 360 degrees. But in physics we keep as many symmetries as are required by the dynamics. As this group gets larger, there is much in the original representation that is no longer invariant; then it appears as if the symmetries do not preserve all the structure there was in the object. But a symmetry of an object is a transformation that leaves the object the same -- identical to itself. So this appearance must be illusory or deceptive: there is nothing to the object except what is invariant. The representation 'clothed' the reality with appearances.

Stated so bluntly, this sophistry will not take in anyone. The correct response is of course to note that a symmetry is a transformation that leaves the object the same in all relevant respects. What are the relevant respects -- what are the inessential aspects, the irrelevant parameters that symmetries can vary -- is equivalent to the question of which transformations are the symmetries. But relevance is contextual. A parameter may be relevant in the solution of one problem and not in another. Two isomorphic groups can differ from each other; they just do not differ as groups, there is no difference if we take only the group operations into consideration. Symmetry, isomorphism, relevant sameness are all context-dependent notions. 
Is radical structuralism coherent?

There is one striking point about the 'ontological position', regardless of whether or not it is cashed out with the concept of invariance. ${ }^{15}$ Instead it must imply: what has looked like the structure of something with unknown qualitative features is actually all there is to nature. But with this, the contrast between structure and what is not structure has disappeared. Thus, once the position is adopted, any difference between it and 'ordinary' scientific realism also disappears. It should, once adopted, not be called structuralism at all! If there is no non-structure, there is no structure either. But for those who do not adopt the view, it remains startling: from an external or prior point of view, it seems to tell us that nature needs to be entirely reconceived, with the appearances classified as pure illusion ${ }^{16}$

This point is not just striking, it is paradoxical. Indeed, as far as my exposition here goes, it comes to us in barely coherent guise. Let me try to suggest a sense for this 'ontological position' (radical structuralism). First, there is the ordinary or old-fashioned way of thinking which has a domain of entities and a family of properties and relations. The properties are instantiated by some entities, the relations by sequences of entities in that domain. We may suppose that the properties and relations are divided into structural and non-structural ones. Some of the properties may of course be described in a rather indirect way -- for example, one structural property might be invariance under certain sorts of operations, such as rigid motions.

Second on the road to abstraction is the representation of all those properties and relations by means of a single structure, a logical space (state space, phase space, ...). When that is done, all the properties and relations instantiated by a given entity or sequence of entities can be summed up by assigning that entity or sequence a location (point or cell) in that space. But this is still not very abstract, for we can then suppose that there are further properties, not present in this representation, which characterize those entities. We might suspect or even insist that there must be such 'hidden variables' if, for example, we notice that more than one entity can have the same location in the logical space. The identity of indiscernibles would imply that there must then be distinguishing unrepresented properties.

So, third step, the radical move: we eliminate the domain of entities in our representation of nature. Instead of saying that entities 17 and 19 both have location q, we say that $\mathrm{q}$ is doubly instantiated. A location in a logical space stands for a complex of properties of all sorts which can together be the complete specification of an individual, in the old way of thinking. Retain the idea that this location is in effect a 
composite property, and allow for instantiation as a primitive notion, a property of properties so to speak. An old-fashioned thinker can accept this as a façon de parler: the statement that COWHOOD is multiply instantiated can just be read as meaning that there are more than one cow, for example. But now comes the 'ontological position', if I understand it correctly: only the multiple instantiation talk (assignment of occupation numbers to the locations or cells of the logical space) is literally accurate. The "there are two ..." way of speaking is not to be taken all that seriously, it is the façon de parler to adopt when speaking with the old-fashioned.

This assertion eliminates the question concerning what might differentiate or individuate distinct occupants of a single cell or location. There are no occupants; there is only multiple occupancy. It is rather easy to see how well this goes with the 'identical particle' problem and one of its solutions -- in fact, Fock space is probably the simplest and most direct scientific illustration of this line of thinking. But there are more venerable precedents in philosophy, under the heading of anti-essentialism. ${ }^{17}$

Now, however, we may have lost touch with concrete reality altogether! There are many familiar examples in which we attribute properties to properties. The statement "Orthogonality is symmetric" and "Orthogonality is invariant under Euclidean transformations" are good examples. Such statements do not imply the existence of anything but abstract entities: properties or relations like orthogonality and properties of properties like symmetry or invariance. So if God had -- so to speak -- decided not to create nature at all, nothing at all that belongs to the proper domain of physics, those statements would still have been true. The statement "X is multiply instantiated", where $\mathrm{X}$ is some property or relation like orthogonality, must be different from this. If God had decided not to create anything concrete, then that statement would have been false. Therefore, taking the contrapositive, if such a statement is true, then there exist entities other than properties and relations. Mutatis mutandis for logical space, configuration space, Fock space .... If the occupation number of any cell or location is more than zero, then there exist entities other than this space. To put it differently, retracing our steps of abstraction: if structure is not just there as mathematical or abstract entity, then it is not true that structure is all there is.

I do not see any way out of this. The radical form of structuralism seems to me to lead right back to reification: the whatever it is that bears this structure may be denied other properties perhaps, but not existence. This does not mean, of course, that there have to be distinguishable particles. What it does mean is 
that we must take as at best metaphorical any attempt to equate particle talk, of any sort, with descriptions of structure. Following Weyl and Wigner, we can say that the classification of elementary particles is via e.g. the irreducible representations of a given symmetry group, or with Mackey via systems of imprimitivity. ${ }^{18}$ But identification via is not identification with!

This discussion is not closed, clearly. ${ }^{19}$ But we' 11 now return from the most radical form to structuralism about science in general.

How is structural realism supported?

As further prolegomenon, we need to look briefly at how Worrall supports his structural realism. This support is, frankly, schizophrenic to empiricist eyes. Worrall needs to give support to three claims. The first is that contrary to the more radical 'revolutions and incommensurability' picture of science, scientific knowledge is cumulative in some important respect. The second is that the knowledge accumulated is of features of the world that transcend the empirical world disclosed in observation and experimentation -features of the reality behind the phenomena. The third and last is that these features constitute structure rather than quality -- that in some significant sense, what we come to know is only the structure of that reality and not what it is like 'in itself', 'intrinsically'.

The air of schizophrenia comes from the fact that all the support given for the first claim is explicitly and admittedly concerned only with an accumulation of empirical knowledge. After a quick survey of the history of optics, Worrall writes ${ }^{20}$

as theories were changing light from chalk to cheese [a British idiom -- $t r$.] and then to superchalk, there was a steady basically cumulative development in the captured and systematised empirical content of optics ... The process at the empirical level (properly construed) was essentially cumulative .... Or take the Newton-Einstein case ... At the empirical level it does seem intuitively reasonable to say that Einstein's theory is a sort of "extension with modifications" of Newton's ... The picture of the development of science certainly seems, then, to be one of essential cumulativity at the empirical level, accompanied by sharp changes of an entirely non-cumulative kind at the top theoretical levels. (Worrall, p. 108-109) 
Very true! Now, what are we to make of that? If we take this at face value, we will not arrive at anything like a scientific realist position.

Worrall notes that himself, saying that these findings tend to lead us "into some sort of either pragmatic or 'constructive' anti-realism". But in opposition to this he now gives the great argument that will support his second claim: that despite appearances, the real accumulation of scientific knowledge is of some reality behind the phenomena:

Such a position [anti-realism, with attention to the accumulation of empirical knowledge] restores a pleasing, cumulative (or quasi-cumulative) development to science ...; but it does so at the expense of sacrificing the 'no miracles' argument entirely. (Worrall, p. 110)

What is this 'no miracles' argument, and how is it supporting the second claim? It is the argument that the success of science at the empirical level would be a miracle. It would be too much of a coincidence or brute fact to be believed, unless we do think that science is pretty well true over all. That is, empirical success would be a miracle unless science was successfully latching onto the truth about what things and processes in nature are like below or behind the part that we can observe, the part that is not displayed in the data but which 'produces' those data.

And what is the support for this contention? Unlike in the first part, no argument here draws on the history of science, details of actual scientific theories, relations between theories, structure of theories, nature of experiment, experimental design, .... It is as if science itself has been forgotten .... Only the scientists have not been forgotten: the support cited lies in the pronouncements of famous scientists in their popular writings, prefaces, and postscripts -- as well as in the expresssions of untutored intuitions putatively found in the common-sensical man in the street. ${ }^{21}$

But didn't appeal to authority disappear from respectable philosophical and scientific reasoning sometime during the Renaissance? This is what I had in mind when I referred to an air of schizophrenia: the tenor and content of argument changes startlingly as we go from the claim of accumulation to the one about observation transcendent reality. 
Finally, what of the third claim, that the truth latched onto concerns solely structural features? We have already seen the two-fold motivation for this amendment which operates equally regardless of the level of description, empirical or theoretical. Today its main impetus comes from the insight that motorized modern mathematics, that the study of invariants, and the reduction modulo contextually irrelevant differences is the short, straight, royal road into the heart of the mathematical object. But in the case we are presently examining, that will fall very short of what Worrall needs. For his claim requires a context-independent, 'objective' division in nature between mere quality and the relational structure in which the qualities appear at the vertices, so to speak. This can be made good only by falling back on a (here unacknowledged) metaphysics in which such an intrinsic-extrinsic distinction makes sense. I rather doubt that today's structural realists in philosophy of science are very anxious to creep into that thicket ... but without that, and without a way of finessing the point, the burden of unacknowledged metaphysics rests heavily on their position.

\section{An Empiricist Structuralism}

I am going to argue that indeed there is a steady accumulation of knowledge in the sciences, and that this knowledge deserves to be called precisely knowledge of structure. But on the view I will present here, there are (both in individual experience and in science) only two sorts of things we deal with directly. These are the concrete, observable things, events, and processes in nature on the one hand and on the other hand, the abstract structures studied in mathematics. We characterize the structure of the former in terms of the latter. To show how this view differs from structural realism and to make it plausible, let us begin with a look at the problem of royal succession in science.

\section{Royal succession in science}

When an older accepted theory falls to its unresolved anomalies, it is still respected as having been a highly successful theory in its time, in the domain where it was applied. In experiment, observation, and application it scored its empirical successes, all of them reasons for its earlier acceptance. The new theory must not only be able to duplicate those successes. We must in addition have an explanation of how and why 
the older theory was in fact so successful. ${ }^{22}$ This is the relationship which took the place of the presumed reduction:

Requirement upon succession. The new theory is so related to the old that we can explain the empirical success of the old theory if we accept the new.

This is a modest requirement, slanted toward empiricist thinking; and below I will defend its modesty against stronger alternatives. I will also give examples to illustrate how the superseding new theory explains those past successes. It is definitely not by explaining how essentially right the old theory was about the underlying structure of nature! It is instead, quite simply, by implying approximately the same predictions for the circumstances in which the older theories were confirmed and found adequately applicable. Thus the past empirical success can now also be counted as an empirical success for the new theory. It is not enough, but it is the initial set of credentials sine qua non for a victory.

If there is a scientific method, this is part of it -- we see here a constraint on new candidates for theory acceptance. And we see an important and theoretically manageable relation that cuts across scientific revolutions. Indeed, we can now identify a body of knowledge which does evolve by accumulation rather than replacement: the empirical knowledge that was tested and is retained, still accepted afterward as triumphs of past science.

So we can reply to Worrall: $Y E S$, there is an accumulation of knowledge through science, but it is knowledge about the observable phenomena. You did make a good guess: there is an accumulation going on throughout all those deep theoretical changes. Moreover this requirement upon theory succession should satisfy the 'No Miracle' intuition! The success of science is not a miracle, because in any theoretical change both the past empirical success retained and new empirical successes were needed as credentials for acceptance. $^{23}$ 
Defence of the empiricist version

It used to be argued that the older successful theory must be shown to be reducible to the new. A good deal of theoretical work was done to perform such reductions, for example of phenomenological thermodynamics to statistical mechanics. But there is no precise, strict sense in which theories are in general reducible to parts of their successors.

The relationship expressed in the above Requirement upon succession is not a requirement of reducibility. The relation is much looser, but nevertheless very demanding. The difference is that it grants conceptual autonomy to the new theory, which is allowed to re-describe nature entirely in its own terms.

Although looser, this relationship admits of rigorous proof, and every advocate of a new theory is at pains to demonstrate it. The loveliest, neatest examples belong to the more mathematical sciences. So, by letting the speed of light in the Special Theory of Relativity go to infinity, you can deduce the relevant Newtonian equations. Literally, that means that Newton's equations are false throughout -- for no finite speed is less than a finite fraction of the speed of light. But it explains, on the basis of the Special Theory of Relativity, why those Newtonian equations performed so well, to such a good approximation, in domains of slow transport over comparatively short time intervals. Similarly if you let the Planck constant go toward zero in quantum mechanical deductions. There is no sense in which models of Newtonian physics can in general be embedded in the models of those newer theories. The transformation groups are too different. But the new theory can explain the empirical rewards earned by the now rejected theory. ${ }^{24}$

There is however also an intermediate candidate for the requirement upon candidates for the successor theory in a scientific revolution. That is Reichenbach's requirement of "continuous augmentation": previously employed principles are assumed to hold approximately, in the limit. ${ }^{25}$ This requirement is precisely modeled on the first especially nice example (Relativity Theory) I just gave. One finds it expressed elsewhere as part of a certain remaining triumphalism in how we might wish to see the past: 
To use a comparison, we could say that creating a new theory is not like destroying an old barn and erecting a skyscraper in its place. It is rather like climbing a mountain, gaining new and wider views, discovering unexpected connections between our starting point and its rich environment. But the point from which we started out still exists, and can be seen, although it appears smaller and forms a tiny part of our broad view gainded by the mastery of the obstacles on our adventurous way up. (Einstein and Infeld, pp. 158-159)

Not at all what Galileo, Bacon, or Descartes wished to say about their forerunners! But more recently Reichenbach's requirement has been embraced by Michael Friedman as inspiration for a renewed Kantian perspective on science. $^{26}$

The difference between Reichenbach's requirement and the above Requirement upon Succession is that the former extends to the entire theory, or parts of the theory still looked upon as having enjoyed empirical success -- as opposed to the empirical successes themselves. It does happen of course that a new theory will resurrect old theoretical principles in some fashion, and not just explain why they managed to reap empirical support. But I see no rationale for this as a stronger requirement.

Let's take an illustrative example: isn't the stronger requirement violated in the transition from Aristotelian to Cartesian or Newtonian physics? Coincidentally this is discussed by Friedman (forthcoming), and he denies the violation, in two ways. First, as a general point, he emphasizes the continuity of theory change, so we have to look at small steps. Relevantly to this he mentions Galileo's postulate of circular inertia as intermediate between Aristotle's laws of motion and the modern. Second, more specifically, Friedman describes how we can see Aristotelian physics as approximately correct in a limited domain described in one particular way: near the earth, in a reference frame with earth at the center.

But it seems to me that there is no such continuous augmentation at the theoretical level -- or rather that, depending on how we construe it, the principle becomes either trivial or clearly false. Appearances to the contrary come from extremely selective attention to certain features of the old theory, whose relevance is only identifiable retrospectively, so as to function in retrospective rationalization. There is a solid core of continuity (perhaps somewhat overstated by Reichenbach and Friedman), namely that the new theory is (ought to be) demonstratively capable of duplicating the empirical successes which we can still see as support 
for the old theory -- at that later time; we need not accept what the old theory advocates saw as support -- and in this way, and this way only, explain why the old theory was as empirically successful as in fact it was.

Clearly the principle of continuous augmentation can be trivially satisfied if we allow any kind of gerrymandered attention to any implications we like, of the old theory, applied to selected features of a roughly characterized suitably small domain of application. If that is allowed there was a process of continuous augmentation leading from medieval astrology to modern medicine, and from Paracelsus to Heisenberg. At least -- this is important -- such a description can then always be cooked up retrospectively.

The more important point, as I see it, is this: in the examples of continuity we can typically distinguish an empirical element which serves as the operative core, from a theoretical aspect which is in fact not retained at all, not even in highly restricted form or in the limit. (There are of course atypical cases, and these are precisely what inspired Reichenbach's requirement upon royal succession in the sciences.)

I'll make this point specific for Friedman's own example. In Aristotelian physics each sublunary body has its natural place, toward which it will naturally move if unconstrained. Any other motion is not 'natural' but 'violent' motion, subject to the principle that nothing moves unless it be moved by something. This natural motion is in a straight line toward the natural place. For the element earth the natural place is the center of the Universe, which is accordingly the center of the Earth. Stones, which are predominantly earth, if released in air or water (whose natural places are above that of earth) above but not too far from the surface of the Earth, will accordingly fall straigt down. That they will do so (to a great degree of approximation, which ignores the gravitational pull of anything other than the Earth) is also implied by Newton's theory. So we see an agreement in a special case, in the limit (of zero contribution to the resultant force on the body by anything other than the gravitational pull of the Earth).

But what is this agreement? It is certainly an agreement on the important empirical prediction of a quite commonly observed phenomenon. This phenomenon did fit very well into Aristotle's theory and provided empirical support for that theory. But if we look at Aristotle's theory, even the very small part that divides motion into natural and violent components, even for a stone near the surface of the Earth, we can immediately see a theoretical conflict with Newtonian physics. The disparity is of course evident only if we pay attention as well to slight modifications in the circumstances. Imagine the stone to be released at the edge of a bowl. It will slide towards the bottom in its natural motion toward the center of the Earth -- 
deflected 'violently' by the resistance of the bowl to penetration. But then it will refuse to continue to display its natural tendency, by leaving the bottom and continuing its slide in an upward direction. This violates the theory, for there is nothing in the situation to which we can attribute violent action upon the stone to account for this. There is no upward push by the bowl. If there were, it would tend to retard the downward slide equally, and -- judging by the diminishing speed upward -- more so nearer the bottom than near the edge. In other words, the natural motion downward would diminish as the stone neared the bottom of the bowl (the valley floor, in a larger case); the larger the bowl, the more noticeably.

It may be objected that I am unfairly switching attention to another situation, in which inertia plays a role as well as gravity. A small part of the Aristotelian theory coincides with a relevant small part of Newton's theory, a larger part fails to do so. But what is this coincidence besides agreement on an observable phenomenon? My example of slightly altered circumstances brings out the fact that the theoretical element (that is, the concept of natural motion and its distinction from violent motion) is entirely foreign to Newton's theory. It is not as if Newton's theory says that in certain restricted cases stones will move rectilinearly toward their natural place. Newton's theory says only that in this restricted case stones behave as if they were moving rectilinearly toward what Aristotle identifies as their natural place. Even that Newton's theory can say only in an extension of its own proper language, in which both theories can be expressed.

Both my example and the objection to it highlight the extreme selectivity that marks such theory comparison. Now I add to this: what guides the selectivity is an interest not in the theoretical relations at all, but rather in the empirical successes which credential the theories. (This is Frederick Suppe's term -- see Suppe 1993; I think he hits the nail on the head.) The new theory needs to establish its credentials as serious contender, and this requires that it can also claim the empirical success which supported the old theory, and which we would not gladly do without. It may achieve this by tailoring itself in the limit and/or to a certain degree of approximation, to certain implications for known sorts of phenomena. But it can also achieve it in a quite different, theoretically novel way. Either is perfectly fine, but only the former illustrates Reichenbach's principle of continuous augmentation.

Finally, perhaps the most important consideration is the following. While the weaker principle/requirement upon succession has a clear rationale, the stronger one can only be proposed on vague general grounds. One can at best cite theoretical conservativeness or calculational advantage, neither of 
which can be more than an inclining consideration. That the new theory must establish its empirical credentials with respect to what we still consider the empirical phenomena successfully handled in the past, that is obvious. But beyond this, what authority does the old theory have, that would entitle it to be respected and even duplicated in any part of its world picture? Reichenbach, as far as I have noticed, speaks only of conservativeness. Friedman, I think, is swayed by the conviction that theoretical transitions, including the most revolutionary, must be guided by a priori principles at a meta-theoretic level.

To an empiricist, this must be ruefully classed as of a piece with other general convictions deriving from the metaphysical instinct -- though an instinct that has been regrettably common also in empiricist ranks. Examples would include the conviction that there must be an inductive logic, or logic of discovery, or other such principles of right reason -- indeed, that rationality must consist in rule-following. This instinct has here retreated to a meta-theoretic level, but is otherwise unchanged.

Structure: an empiricist view

Worrall made a second guess: that what is retained through theory change is structure. Such assertions as that there are mechanical atoms or a plenum, caloric or phlogiston, ether or Rutherford atoms -these are not retained. But something of the structure of the old scientific image goes over to the new, and the key word is structure. As almost every commentator has somewhat sadly remarked, this key word has its own problems. What exactly is the difference between matter and form, content and structure? Aren't such distinctions painfully context-dependent? Is there really an objective difference in nature, as opposed to merely in our representations of nature? Is it not a little embarrassing to start with the thesis that what is preserved through scientific revolutions is the structure attributed to nature, and then to have to identify structure by noticing what has been preserved? Must this philosophy of science ultimately rest on a metaphysics to distinguish intrinsic and extrinsic properties (essences and accidents, substantial form and prime matter, relation and pure quality)?

But we can look at the matter quite differently, and prevent ourselves from sinking into this metaphysical morass that swallows all seekers for the true foundations of being. The empirical successes of the older theories were partial successes of a very distinct sort: their representations of nature, the models they 
made available for representation of the observed phenomena, were partially accurate. These successes consisted in their success of fitting the data, the deliverances of experimental and observational experience. There was something they got right: the structure, at some level of approximation, of those phenomena. Here the word "structure" is used to point specifically to a certain character, defined by certain measurable parameters both old and new theory use to describe those empirical successes.

Just look at those empirical phenomena! They have, in an intuitive sense, both structure and intrinsic qualities, it seems. What the intrinsic qualities really are, each new theory has something to say about. Colors, for example, first accepted as qualities of light rays by Newton, are later described in terms of wavelength. But there are a good many 'low level laws' which take the form of simple equations, describing the structure of those phenomena. These are closely connected with the empirical successes that every succeeding theory will have to duplicate, at least by approximation in a limited domain. These phenomenal structures must fit, in a certain way, into the new theoretical models. The laws of reflection and refraction of geometric optics, the laws of Archimedes, the laws of inertia, of free fall, of the pendulum -- these are all simple mathematical descriptions of certain aspects of the phenomena. They are not retained in their early precise and unrestricted form. But they are retained as the structure phenomena take when observed at a certain level of discernment. ${ }^{27}$ We can plausibly think of the empirical description as the stable evolving surface structure of science on the face of a radically, rapidly altering theoretical content within.

Synoptically then: there are just two realms of scientific investigation, hand in hand by experimentalists and theorists. On the one hand there are the phenomena which are investigated. On the other hand there are the models, abstract structures studied in mathematics, which the theory advances as representations of those phenomena. The representation is always partial and selective. You and I are mechanical systems, that is, we are correctly represented by certain mechanical models. But however good those models are, they omit quite a lot about us. Since those models considered in their own right are mathematical structures, they are known only in the way things are known mathematically. In mathematics, things are described only up to isomorphism -- it makes no sense there to speak of differences between isomorphic structures -- and that is why it makes perfect sense to say that here we are dealing solely with structure. 
True, the distinctions are context-dependent; so is the very word "isomorphism", and even more so is the selection of "relevant" parameters for theoretical description. But by becoming comfortable with this context-dependence we make sense of the intuition that science presents us with the structure, and that it is knowledge of the structure of the empirical phenomena which is accumulated. For what that means now is that there must be mathematically describable relationships between the new and the old models which pertain in the required way to just those measurable parameters in which the older empirical successes were couched. To put it briefly:

Science represents the empirical phenomena solely as embeddable in certain abstract structures (theoretical models), and those abstract structures are describable only up to structural isomorphism. $^{28}$

There is warrant for the assertion of an accumulation of empirical knowledge through theory change precisely if it can be demonstrated for phenomena counted among the empirical successes of earlier science that, if they are embeddable in the new models then they are 'approximately' embeddable in the old models. ${ }^{29}$

This empiricist re-construal is scant comfort to the scientific realist, of course. It also sets aside as unimportant the conceptual puzzles about how to distinguish structure from content or quality, which beset socalled structural realism. But it provides a balanced view of scientific theory change, taking some of the mystery out of scientific revolutions. All it takes, to achieve this more balanced view, is to dispel the lazy illusion that we could do this by means of the simple expedient of either reifying the models or regarding them as delineating the objective structure of a hidden qualitative content.

\section{BIBLIOGRAPHY}

Note: Original publication dates are important for the historical parts of this paper; an entry of form "Hertz, Heinrich. 1893/1962 Electric Waves. Tr. D. E. Jones, NY Dover." Indicates that the book was originally published in 1893, but citations are from the 1962 Dover edition. 
Blackmore, J. ed. 1992. Ernst Mach -- A Deeper Look. Boston Studies in the Philosophy of Science, vol. 143. Dordrecht: Kluwer.

Boltzmann, Ludwig. 1905a. "Theories as representations", translation of Boltzmann 1905b, 253-269 in Danto and Morgenbesser, 245-252.

Boltzmann, Ludwig. 1905b. Populaere Schriften. Leipzig: J. A. Barth.

Bueno, Otavio. 1999a. "What is structural empiricism? Scientific change in an empiricist setting". Erkenntnis 50: pp. 59-85.

Bueno, Otavio. 1999b."Empiricism, Mathematical Change and Scientific Change", forthcoming in Studies in History and Philosophy of Science.

Bueno, Otavio 1999c. "Empiricism, Conservativeness and Quasi-Truth" Philosophy of Science 66: S474-S485.

Cassirer, Ernst 1923. Substance and Function and Einstein's Theory of Relativity. Tr. W. C. and M. C. Swabey; Chicago: Open Court.

Castellani, Elena 1998a. "Galilean particles: an example of constitution of objects", 181-194 in Castellani $1998 b$.

Castellani, Elena ed. 1998b. Interpreting Bodies: Classical and Quantum Objects in Modern Physics. Princeton: Princeton University Press.

Coffa, Alberto "From geometry to tolerance: sources of conventionalism in nineteenth-century geometry", in Colodny 1986, 3-70.

Colodny, R. G. 1986. From Quarks to Quasars. Philosophical Problems of Modern Physics. Pittsburgh: University of Pittsburgh Press.

Da Costa, N. C. A. and French, S. 1990. "The model-theoretic approach in the philosophy of science", Philosophy of Science $57: 248-265$.

Danto, Arthur and Morgenbesser, S. eds. 1960. Philosophy of Science. New York: Meridian Books. Einstein, Albert and L. Infeld. 1938. The Evolution of Physics. New York: Simon and Schuster. 
Fine, Arthur. 1998. "The Viewpoint of No-One in Particular" . (Presidential Address, Central Division, 1998). Proceedings of the American Philosophical Association 72 : 9-20.

French, P. et al. eds. 1979. Midwest Studies in Philosophy; vol. iv, Metaphysics. Minneapolis: University of Minnesota Press.

French, Steven. 1998. "On the withering away of physical objects", in Castellani 1998b, 93-113.

Friedman, Michael. "Transcendental philosophy and a priori knowledge: a neo-Kantian perspective", forthcoming in a volume on a priori knowledge, ed. P. Bhoghossian and C. Wright.

Ghins, Michel. 1992. "Scientific realism and invariance", Philosophical Issues 2: 249-262.

Hertz, Heinrich. 1893/1962 Electric Waves. Tr. D. E. Jones, NY Dover.

Hertz, Heinrich 1894/1956. The Principles of Mechanics tr. D. E. Jones and J. T. Walley, New York: Dover. Jammer, Max.1964. The Concept of Mass. Cambridge, MA: Harvard University Press, 1961; reprinted New York: Harper and Row.

Ladyman, James. 1998a. "Structural realism: epistemology or metaphysics?", Studies in the History and Philosophy of Science 29A: 409-424.

Ladyman, James. 1998b. Structural Realism and the Model-Theoretic Approach to Physical Theories. Dissertation, University of Leeds.

Mach, Ernst 1910. "Sensory elements and scientific concepts", tr. J. Blackmore, in Blackmore 1992.

Maxwell, J. C. 1881. Elementary Treatise on Electricity. Oxford.

Mermin, N. D. 1998. "What Is Quantum Mechanics Trying to Tell Us?" American Journal of Physics 66: $753-767$.

Nozick, Robert. 1998. "Invariance and objectivity" (Presidential Address, Eastern Division, 1997). Proceedings of the American Philosophical Association 72: 2148.

Planck, Max. 1909/1992. "Die Einheit des physikalischen Weltbildes", Physikalishes Zeitschrift 10 (1909), 62-75; tr. of section 4 in Blackmore 1992.

Poincaré', Henri. 1905/1952 Science and Hypothesis. New York, Dover Publications.

Psillos, Stathos. 1995. "Is structural realism the best of both worlds?" Dialectica 49: 15-46. 
Reichenbach, Hans. 1921/1959. "The present state of the discussion on relativity", pp. 1-45 in Reichenbach 1959; translation of "Der gegenwaertige Stand der Relativitaetsdiskussion" (1921); reprinted in Reichenbach 1979, vol. 3, pp. 342-403.

Reichenbach, Hans. 1959. Modern Philosophy of Science. Tr. M. Reichenbach; London: Routledge and Kegan Paul.

Reichenbach, Hans. 1979. Gesammelte Werke. Ed. A. Kamlah and M. Reichenbach; Braunschweig: Vieweg. Russell, Bertrand. 1912/1959. Problems of Philosophy. Oxford University Press, 1959.

Russell, Bertrand. 1927. The Analysis of Matter. London: Allen and Unwin.

Stalnaker, Robert. 1979. "Anti-essentialism" in French et al., 343-355.

Stein, Howard. 1989. "Yes, but ...-- Some skeptical remarks on realism and anti-realism" Dialectica 43: 4765.

van Fraassen, Bas C. 1989. Laws and Symmetry. Oxford University Press.

van Fraassen, Bas C. 1991. Quantum Mechanics: an Empiricist View. Oxford University Press.

van Fraassen, Bas C. 1993. "From vicious circle to infinite regress, and back again"; pp. 6-29 in D. Hull, M.

Forbes, and K. Ohkruhlik (eds.) PSA 1992, Vol. 2. Evanston: Northwestern University Press.

van Fraassen, Bas C. 1997. "Structure and perspective: philosophical perplexity and paradox", pp. 511-530 in M. L. Dalla Chiara et al. (eds.) Logic and Scientific Methods. Vol 1. Dordrecht: Kluwer.

Weyl, Hermann. 1952. Symmetry. Princeton: Princeton University Press.

Weyl, Hermann. 1931/1950. The Theory of Groups and Quantum Mechanics. $\quad$ tr. H. P. Robertson, New York: Dover Publications.

Wilson, Catherine. 1995. The Invisible World: Early Modern Philosophy and the Invention of the Microscope. Princeton, N.J. : Princeton University Press.

Worrall, John. 1989. "Structural realism: the best of both worlds?" Dialectica 43: 99-124.

\section{NOTES}

${ }^{1}$ I wish to acknowledge gratefully the inspiration of Arthur Fine's "The Viewpoint of NoOne in Particular", and also my debt to Otavio Bueno and James Ladyman, who kindly allowed me to take up their unpublished work in my seminar (fall 1998), and to Anja 
Jauernig for much helpful discussion and correspondence. An earlier version was delivered as "Structure, Realism, and Reality" at the University of Leiden June 1999, and I owe much to the audience discussion there.

${ }^{2}$ When Poincare later says that Maxwell had shown that there must exist mechanical models of electromagnetism, he presumably thought that this subsumption under the generalized Lagrangian mechanics was successful. As we shall note below, this already derives its sense at best from several successive weakenings of what can count as a mechanical model.

${ }^{3}$ Note however the vagaries of linguistic change in science. Stein makes a good case for holding that the retention of the word "atom" and discarding of "ether" was historically arbitrary: "our own physics teaches us that there is nothing that has all the properties posited by nineteenth-century physicists for the ether or for atoms; but that, on the other hand, in both instances rather important parts of the nineteenth century theories are correct." op. cit. p. 57

${ }^{4}$ For these sources and an illuminating discussion see Wilson 1995, pp. 57-65, 218-220. ${ }^{5}$ While Descartes' contribution is an original reconception of the physical world, this dream of so constructing the uniquely intelligible world was part of the Zeitgeist; compare e.g. Hobbes' De Corpore.

${ }^{6}$ It is because they have embraced the mathematicians' insight that to cut to the chase, one needs to study the invariant structure underlying apparent or surface differences and variations. But the mind recoils a bit from this level of abstraction, and it is not easy to convey in concrete terms. So then the temptation to metaphysics sticks up its two heads again, with their two simplistic panjandrums to give this development a gloss of familiarity. One head suggests we simply reify, and say that the mathematical structure which captures those invariants is a picture of what the world is really like. The other head says well, no, the invariant structure 
thus represented is the structure of something real, a true je ne sais quoi of course, which has its own unknown and unknowable qualititative character as well. When the thirst for explanation can't be slaked by anything real, it will take a verbal gloss or empty metaphysical form as its drink. Howard Stein, speaks similarly of "judgement ... seduced by the great desire to feel that it understands" and of "a relaxation of intellectual standards under ... ideological pressure" when 'explanations' are accepted although in fact they have no explanatory value at all (op. cit. p. 55).

${ }^{7}$ To speak of Maxwell's Theory is somewhat ambiguous. In his later monograph (1881) he tried for a 'deduction from the phenomena' with no reliance on any ideas about the medium that carries electromagnetic waves -- this leads some to think of his theory as a 'no ether' theory well before such experiments as Michelson and Morley's provided strong empirical reasons against the reality of the ether. Through the generalized Lagrangian formalism, explicit reference to the ether is eliminated. But none of this warrants the conclusion that a far-reaching conceptual revolution (needed to allow that a complete good theory of these phenomena could take a form other than a mechanical ether theory) had already occurred.

${ }^{8}$ The answer is, I think, that what is transparent to the mind is the mathematical structure theoretical science creates, and what is understood in addition is how, entirely contingently and a-rationally, this structure applies in experience to the phenomena within our ken. But I will come back to that below.

${ }^{9}$ Bueno (forthcoming) points out quite correctly that the debate between scientific realists and empiricists concerning the no-miracle argument is inescapably fruitless under certain conditions. For the main part, scientific realists insist on the centrality of the demand for explanation; the empiricist does not recognize this demand in general. Indeed, the primacy of this demand for explanation is characteristic of the sort of metaphysics with which the 
empiricist tradition has always been at odds. Common concern with the rationality of scientific change may take us out of this impasse.

${ }^{10}$ I want to thank Gideon Rosen for a helpful, truly clarifying discussion of what Ladyman calls the ontological position.

${ }^{11}$ See Psillos 1995 for the arguments that in fact no sense can be made of these dichotomies, and the conclusion that structural realism consistently pursued leads to a standard variety of scientific realism.

${ }^{12}$ For details on this episode in the philosophy of quantum mechanics, see my 1991 , Chapters 11 and 12; for instructive articles by Schroedinger and Heisenberg, as well as contemporary contributions, see Castellani 1998b. French 1998 brings out very clearly the underdetermination of metaphysics by physics, most saliently visible in this example, and its role in motivation for more radical forms of structuralism as metaphysically frugal response.

${ }^{13}$ Beside Ladyman's discussion we have also recently two presidential addresses is division of the American Philosophical Association on this topic: Arthur Fine, "the Viewpoint of No-One in Particular" (Central Division, 1998) and Robert Nozick, "Invariance and objectivity" (Eastern Division, 1997). For an earlier discussion, see e.g. Ghins 1992. ${ }^{14}$ Arthur Fine (1998) distinguishes these concepts relating to objectivity very carefully, separating them moreover from procedural or methodological objectivity, as well as from the more extravagant philosophical notions of the 'view from nowhere' (Thomas Nagel) and the 'absolute conception of the world' (Bernard Williams). The transition from procedural objectivity to such moves in ontology are reminiscent of the transition from 
Galileo's methodological discipline with respect to 'primary' qualities to a reductionist ontology in which all but primary qualities lose their 'objective' significance.

${ }^{15}$ I have some doubts about precisely how the invariance enters. The ontological position is meant to include a statement about nature, but Layman's discussion shifts from structure in nature to the structure of the scientific theories. Symmetry arguments, requests for covariant or coordinate-free formulations, and the like are important guides to theorizing. But it is not immediately obvious what those considerations show us about the world described by those theories. That is precisely the question which a more elaborate Ladyman variation on structuralism would need to answer.

16 The last chapter of Ladyman's dissertation and his ending quote from Stein (that the crucial relation to focus on is that between the 'Forms' (mathematical representations) and the phenomena) suggest that the Ladyman variant may be closer to the empiricist structuralism outlined below than to Worrall's. But Ladyman adds certain other metaphysical theses, such as realism about modality and about abstract entities which I would hope to avoid.

${ }^{17}$ For a more extended discussion see my 1991, Chapter 12; for the logical underpinnings see also Stalnaker 1979.

${ }^{18}$ For an introduction and discussion of these approaches see Castellani 1998b, Introduction, and Castellani 1998 b.

${ }^{19}$ The extreme logical idealism (in which nothing concrete retains independent reality) which I implicitly discounted in my argument was perhaps actually Cassirer's final position. (Thanks to Anja Jauernig for a vivid description.) In 1921 Reichenbach praised Cassirer's neo-Kantian interpretation of relativity theory, including its consequences for what "truth" means for natural science, in terms that he must have greatly regretted later on (see Reichenbach 1959, p. 28 or Reichenbach 1979, p. 374). 
${ }^{20}$ Speaking against the radical 'revolution' view associated with Kuhn he adds in a footnote: "Genuine examples of 'Kuhn loss' of captured empirical content are remarkably thin on the ground -- provided, that is, that empirical content is properly understood." (Note 15, p. 108)

21 Poincaré's remarks cited from his Science and Hypothesis (Worrall pp. 117-118) are scanty, and not precisely unambiguous. It is possible, as I showed above, to find other inspirational texts in the history of science, and of course also to cite such philosophers as Bertrand Russell and Grover Maxwell.

${ }^{22}$ In general, empiricists will not accept demands for explanation among criteria of adequacy for any account, whether of the world, of historical/social phenomena, or of success in any enterprise, practical or intellectual. But this is a reservation to be kept so as to pre-empt metaphysical extravagance. In the present context, the explanations under discussion consist precisely in a demonstration that the empirical predictions implied by the old theory must be approximately correct for certain kinds of cases, on the assumption that those of the new theory are precisely correct. It is natural to use the term "explanation" for such demonstrations, and acknowledging this requirement does not imply the more general sorts of demands for explanation that tend to obstruct the realistempiricist debate in general.

23 As the attentive reader will have noticed, I honor the 'No Miracles' intuition by answering a question subtly different from the one the realist reads into it. My question is: how can we account for the continuous increase in the empirical success of science? Realists attempt instead to answer: what explains the empirical success of science ueberhaupt? Moreover, they understand this question in such a way as to demand an ontology, thus addressing, to my mind, a question which does not arise at all. 
${ }^{24}$ Bueno (forthcoming) presents a precise form of the sort of requirement upon succession which is under discussion here. I have benefited from Bueno's treatment of this subject. However, the precise form Bueno favors is framed in the partial structures approach (Da Costa, French, et al). The location of structure on the side of the mathematical representations of the phenomena aligns the partial structures approach here with various forms of the so-called semantic or model-theoretic approach to science. I am presently not linking my discussion here to either this or any other formal explication of the requirement. 25 "Verfahrens der stetigen Erweiterung", Reichenbach 1979, p. 361; the phrase is misleadingly translated as "method of successive approximations" in Reichenbach 1959, p. 38. I thank Anja Jauernig for drawing my attention to this crucial part of Reichenbach's view.

${ }^{26}$ I draw here on the Hempel Lectures (delivered by Friedman at Princeton University, April 1999) and on Friedman (forthcoming).

${ }^{27}$ This could be put into more structuralist sounding language, characteristic of the semantic view of theories. The mathematical structure of a data model is to be related, in the way indicated, to those mathematical structures -- the theoretical models -- which the theory provides.

28 What about the relation between phenomenon and mathematical structure -- what is the status of statements that assert specific phenomena to 'fit into' a specific structure? This is the topic which Logical Postivists tried to handle with their idea of privileged 'observation statements'. I have developed an alternative account, as yet presented somewhat disjointedly in two parts: see my 1993, parts 1 and 3, and 1997, part 3. 
${ }^{29}$ The phrase "approximately embeddable" needs explication of course. Like "isomorphism" it is context dependent; there must be a contextual determination of what counts as relevant conditions and relevant degrees of approximation. With these contextual factors fixed, I construe an approximate embedding in structure $\mathrm{S}$ to be simply an embedding in a structure $\mathrm{S}^{\prime}$ relevantly like $\mathrm{S}$. But see the earlier reference to Bueno for an alternative approach. 